\title{
On the definition of an ideal amorphous solid of uniform hard spheres
}

\author{
Long-Thang To ${ }^{a}$, Daryl J. Daley ${ }^{\mathrm{b}}$, Zbigniew H. Stachurski ${ }^{\mathrm{c}, *}$ \\ ${ }^{a}$ Center for Science and Engineering of Materials, Australian National University, Canberra, ACT 0200, Australia \\ b Centre for Mathematics and its Applications, MSI, Australian National University, Canberra, ACT 0200, Australia \\ ${ }^{\mathrm{c}}$ Department of Engineering, FEIT, Australian National University, Canberra, ACT 0200, Australia
}

Received 12 December 2005; accepted 8 February 2006

Available online 2 May 2006

\begin{abstract}
Perfection of structure is defined firstly by the definition of imperfections that may occur in that structure, and secondly by the strict requirement of absence of those imperfections. An ideal amorphous solid is a geometrical structure with perfectly random (as distinct from disordered) packing of spheres/atoms. This is achieved by requiring all spheres to be in fixed positions (no rattlers) and the packing to obey certain statistical rules (without exceptions). The random configurations of local clusters are described by the mathematics of self-avoiding random walks, and the distribution of mutual contacts (coordination numbers) is described by combinatorics developed in connection with an earlier work on the structure of liquids. Flaws in the structure are defined. An ideal amorphous solid, based on packing of identical spheres and without any flaws, appears to have packing density close to approximately 0.61 . Flaws which form clusters with close packing configurations (fcc and hep) have the effect of increasing the packing density, whereas other type of flaws, i.e., loose spheres or vacancies will inevitably decrease the packing density. This relationship is revealed by analysis of recently published experimental packings and computer simulations. In that sense, the ideal amorphous solid described here is entirely new and original.
\end{abstract}

(c) 2006 Elsevier SAS. All rights reserved.

Keywords: Touching spheres; Cluster configuration; Packing imperfections

\section{Introduction}

The classical science of crystallography describes ideal crystalline solids by emphasising their perfection, which is defined by common usage as the state of total absence of imperfections. Then, defects in crystalline solids are defined with regard to the perfection of the ideal crystalline structures. Defects introduce disorder in ideal crystalline solids and alter their behaviour. Many properties of real crystalline solids (both natural and synthetic materials) are governed directly by the presence of defects in their structure. For example:

- Diffusion is governed by the density of vacancies or interstitials (point defects) [1].

- Screw dislocations in zeolites can confine diffusion to one direction only (line defects) [2].

\footnotetext{
* Corresponding author. Tel.: +61 26125 5681; fax: +61 261250506.

E-mail address: zbigniew.stachurski@anu.edu.au (Z.H. Stachurski).
}

- Plastic deformation is understood in terms of dislocation glide (line defects) [3], or grain boundary sliding (surface defects) [4].

- Semiconductors are created by doping crystals (introducing defects) to modify their electronic structure (point defects) [5].

The significance of these relationships is reinforced when realising that no diffusion is possible without defects, that crystals reach their theoretical strength without yielding if no dislocations are present, and that semiconductors become insulators if doping is removed.

In the world of natural and synthetic materials there are also real amorphous solids whose structure is frequently, but erroneously, considered to be at the limit of disorder of the corresponding crystalline solid. Yet it is not intended that amorphous should mean defective, but rather random. Our knowledge of amorphous structures seems incomplete when compared with that of crystalline solids. In particular, the concept of an ideal amorphous solid and the corresponding theory are lacking. We 


\begin{tabular}{llll}
\hline Crystalline solids & & Amorphous solids & \\
\hline ideal crystalline & real crystalline & real amorphous & ideal amorphous \\
solids & solids & solids & solids \\
theory of & imperfections & imperfections & theory of \\
crystallography & (defects) & (flaws) & amorphousness \\
perfect order & disorder & imperfect & perfect \\
& & randomness & randomness \\
\hline
\end{tabular}

Fig. 1. A diagram of the solid state area, divided into the crystalline and amorphous fields, and sub-fields with corresponding descriptions and underlying theories. The sub-fields with italic font delineate solid state research approached in this paper from a new perspective.

propose that ideal amorphous solids should be defined first and then, by analogy with crystalline materials, real amorphous solids will be found to possess imperfections, defined relative to the ideal amorphous structure and not with regard to any assumed crystal lattice. These imperfections should be found to influence the properties and behaviour of real amorphous solids. So, a general view of condensed matter can thus be represented by the diagram shown in Fig. 1 (for the sake of generality quasicrystalline materials are included under crystalline solids).

Although the fields on the left hand side of the diagram are well established and understood, yet those on the right-hand side pose several questions - can ideal amorphous solids be defined and how? The answer is tentatively affirmative-this paper describes in some detail at least one class of an ideal amorphous structure based on packing of spheres of the same size with a distribution function for the arrangements of clusters of touching spheres, which are considered to be the building blocks of the amorphous body. We believe that the ideal amorphous solid presented here is original. It is based on configurations of clusters defined separately from the distribution of coordination numbers, and that this family of distributions is necessary to define the structure. What other conditions would make it sufficient is an open question.

The classical study of the solid state has foundations laid deep in the concept of order of the crystalline state. Very briefly, the idea of construction of crystalline bodies by regular packing of identical balls (Haüy 1780) lead to theoretical developments of the Bravais crystal lattices (Bravais 1890) and point and space group symmetries (Schönflies 1891). By comparison, a theory of ideal amorphous structures must be considered to be in its early stage of development. It is possible to separate the study into science dealing purely with the geometry of the atomic packing in the solid body, and another part concerned with atomic interactions and thermodynamics. The approach followed here is to assume that atoms can be represented by spheres and then define ideal solids considering geometrical properties only, without engaging thermodynamic forces and constraints at this stage. Thermodynamic studies should come later, usually verifying or falsifying the proposed structures. Neither molecular dynamics simulations of amorphous solids (which inevitably lead to structures containing imperfections as we intend to show later) nor the study of real amorphous materials can distinguish at this stage between the ideal and the real states, mainly because there is no clear awareness of the need for the former.

In the following sections of the paper we continue to develop an elementary theory for ideal amorphous solids based on the principle that any structure, which obeys given laws without exception, is ideal. In particular, we delineate the process for the description of an ideal amorphous solid, and develop statistical rules that the structure must obey without exception to qualify as the perfect solid. This is followed by a definition of imperfections that are possible in an ideal amorphous structure, accompanied by an analysis of published experimental data corroborating the effect that imperfections have on the packing density of the body. The definition of imperfections in ideal amorphous structure opens a new interpretation of the elusive maximum random close packing of spheres, supposedly close to 0.64 [6]. At the end we show that imperfections can be the cause of increasing density of packing, and that physical packings of spheres approaching that density are artefacts of gravity and friction.

\section{Random packing of non-intersecting touching spheres}

From the geometrical point of view, an ideal amorphous body can be represented by a number $N$ of randomly packed touching but non-intersecting spheres of identical diameters in a compact convex region, where $N$ is as large as may be necessary for a meaningful statistical analysis, and satisfying conditions of translational and rotational invariance (frame independent, isotropic body). We assume the existence of such a body and take it to be self-evident that maximally jammed packing of the spheres is necessary [7] so that it is a rigid solid. For the ideal amorphous solid, to be described here, we impose a stricter condition on the packing such that all spheres are in fixed positions and loose spheres are entirely disallowed.

The sphere is fixed by surrounding touching spheres if it cannot be moved within the cluster without disturbing its neighbours. This condition implies that the touching spheres form a cage around the inner sphere. In $\mathbb{R}^{3}$ a minimum of 4 outer spheres is needed to fix the inner sphere [8]. Otherwise the inner sphere is loose, and can move [9].

Let $S_{a}$ represent a sphere of diameter, $a$, and let $X$ be a set of points in Cartesian space representing the centres of the $N$ spheres,

$X=\left\{x_{1}, x_{2}, \ldots, x_{N}\right\}$.

Then, $S_{a}+X$ is a packing of spheres of the ideal amorphous solid. Since the collection of spheres is defined uniquely and precisely by their centres and diameters, the definition of the structure of an ideal amorphous solid rests entirely with the description of the set $X$. We first note a property of scale invariance: if $S_{a}+X$ is a packing for an ideal amorphous solid, then so is $S_{c a}+c X$ for every positive $c$. We can now assert some essential properties required of the set $X$ :

2.1. For any point, $x_{\ell} \in X$, its nearest neighbours are always equidistant from that point, i.e., $\left|x_{\ell j}-x_{\ell}\right|=a$ for $j=1, \ldots, \kappa\left(x_{\ell}\right)$, where $\kappa\left(x_{\ell}\right)$ is the number of nearest 
neighbours. This requirement reflects the conditions that spheres are non-intersecting, that all spheres have the same diameter, and that nearest neighbours are touching.

2.2. The point, $x_{\ell}$, must always lie inside a polyhedron defined by the nearest neighbours, $x_{\ell j}$, as its vertices. This condition requires that, with $\kappa\left(x_{\ell}\right)=k$ : for $4 \leqslant k \leqslant 9$ no more than $k-1$ contacts must be located on one hemisphere in order to maintain the sphere in fixed position, for $k \geqslant 10$ the sphere is always in a fixed position.

2.3. The number of nearest neighbours, $\kappa\left(x_{\ell}\right)$, is bounded: $4 \leqslant \kappa\left(x_{\ell}\right) \leqslant 12$. The lower limit of 4 is imposed by the condition that all spheres are fixed [9], and the upper limit of 12 is established as an absolute limit by the GregoryNewton theorem $[10,11]$.

We propose that if a monoatomic amorphous solid is an ideal amorphous solid (IAS) then its structure must conform without exception to two statistical properties, subject to invariance under rotation:

- $\Phi_{k}\left(v_{1}, \ldots, v_{k}\right), k=4, \ldots, 12$, is a family of joint probability density functions, on $k$ unit vectors $v_{1}, \ldots, v_{k}$ in $\mathbb{R}^{3}$, of the configurations of clusters of spheres.

- $\Psi\left(\kappa\left(x_{\ell}\right)\right), k=4, \ldots, 12$, a probability distribution for the number of nearest neighbours (coordination number).

\section{Configurations of random clusters}

\subsection{Contact points of a sphere}

Suppose we regard clusters of touching spheres as being the building blocks of amorphous solids, analogous to a unit cell of a crystal. A local cluster of $k+1$ impenetrable spheres, all of the same diameter $a$, comprises an inner sphere, with centre $x_{\ell}$ say, which is touched by exactly $k$ outer spheres with centres at $x_{\ell j}, j=1, \ldots, k$. Subject to this contact requirement, the local cluster can have any configuration, regular or otherwise. Where needed we denote the number of contact spheres in the local cluster with centre $x_{\ell}$ by the function $\kappa\left(x_{\ell}\right)$. Any sphere can belong to several clusters.

For each local cluster we identify the contact points between the inner and outer spheres by radial vectors, $\overrightarrow{\mathbf{R}}_{j}, j=1, \ldots, k$, drawn from the centre of the inner sphere to the contact points on its surface as shown in Fig. 2(a). The contact points bisect the distances from the centre of the inner sphere to the centres of its nearest neighbours. There are three general properties related to these vectors:

3.1.1. The magnitude of each vector is equal to half the sphere diameter,

$$
\left\|\overrightarrow{\mathbf{R}}_{j}\right\|=\frac{1}{2} a
$$

3.1.2. The angle, $\phi\left(R_{i}, R_{j}\right)=\operatorname{arcos}\left(\left\langle R_{i}, R_{j}\right\rangle / \frac{1}{4} a^{2}\right)$, between any two vectors emanating from the centre of the sphere cannot be less than $\frac{1}{3} \pi$, and in general,

$$
\frac{1}{3} \pi \leqslant \phi\left(\overrightarrow{\mathbf{R}}_{i}, \overrightarrow{\mathbf{R}}_{j}\right) \leqslant \pi \quad(1 \leqslant i<j \leqslant k)
$$

3.1.3. The number of vectors (contact points) for each sphere is:

$$
4 \leqslant \kappa(x) \leqslant 12
$$

The last two functions, $\phi\left(R_{i}, R_{j}\right)$ and $\kappa\left(x_{\ell}\right)$ are statistical variables of the local clusters.

Note that a sphere centred at $x_{\ell}$ and its surface contact points define the configuration of a local cluster uniquely and sufficiently. From now on we shall focus on the properties of the sphere alone, but bear in mind that it represents the whole cluster. Thus, the configuration of contact points on the sphere can be described in terms of a vector polygon with vertices $R_{j}$, de-
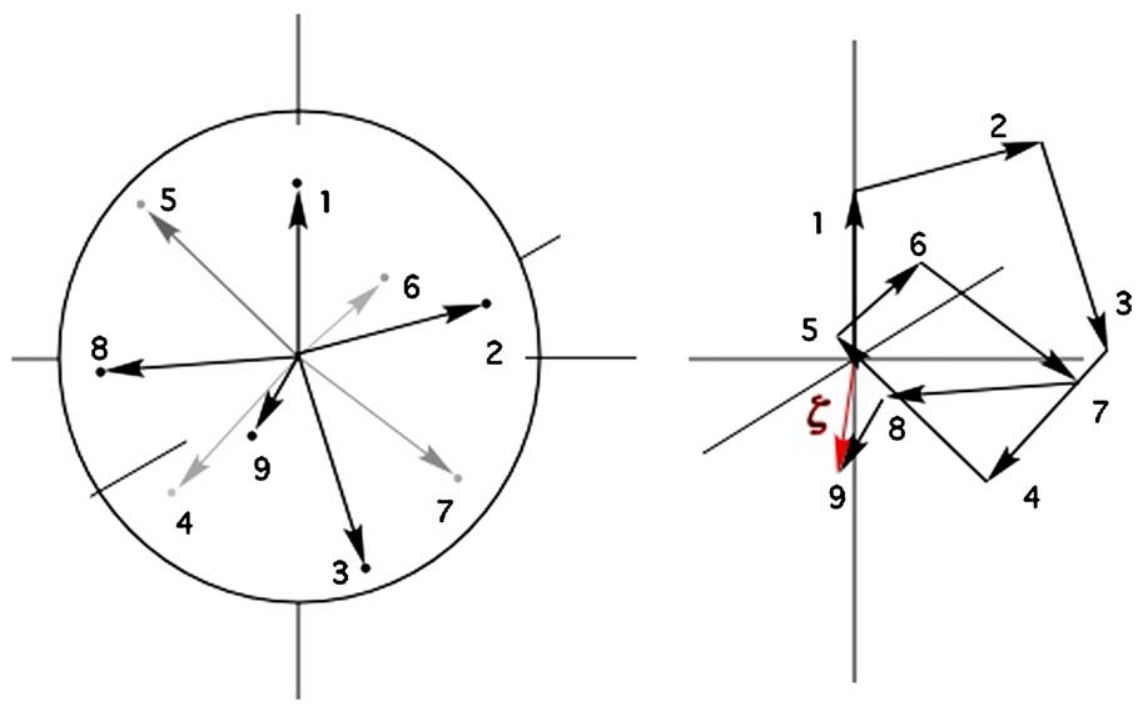

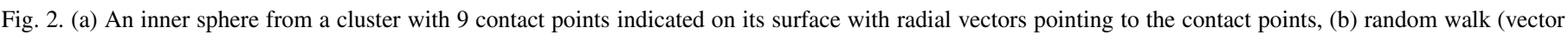
polygon) created by the radial vectors with $\zeta$ as the closing vector. 
fined by:

$\sum_{j=1}^{\kappa\left(x_{\ell}\right)} \overrightarrow{\mathbf{R}}_{j}-\vec{\zeta}=0$.

The sum represents a chain of the radial vectors and the second term is the closing vector of the 3-dimensional polygon. Fig. 2(a), (b) shows an example of an inner sphere with 9 random contact points and the corresponding vector polygon. We also define a function, $\zeta$, equal to the magnitude of the vector, $\vec{\zeta}$ :

$\zeta=\|\vec{\zeta}\|$

$\zeta$ is a summary statistic of any particular local cluster. It should suffice to state that for any number and arrangement of contact points, the $\zeta$-function assumes values in a finite interval:

$0 \leqslant \zeta \leqslant \zeta_{\max }\left(\kappa\left(x_{\ell}\right)\right)$

For each value of the number $k=4, \ldots, 12$, there is a unique maximum value of the $\zeta$-function $=\zeta_{\max }(k)$. It can be imagined as corresponding to an arrangement in which the outer spheres are rolled on the surface of the inner sphere towards the south pole into a close packed arrangement. The values of $\zeta_{\max }(k)$ have been calculated according to this approach, and are listed in Table 1. If the further condition is imposed requiring that the inner sphere be fixed by the outer spheres, then the values of $\zeta_{\max }(k)$ change correspondingly to $\zeta_{\max }^{\text {fixed }}(k)$, as shown in Table 1 , third column. These values were calculated assuming that one of the outer spheres moves to a position diametrically opposite another touching sphere, so that for $k=4$ we have only two radii (separated by an angle $\frac{1}{3} \pi$ ) contributing to the value of $\zeta_{\max }^{\text {fixed }}(4)$, for $k=5$ only three, and so on (the actual values must be arbitrarily smaller since the sphere has to be on the other hemisphere, however imperceptibly). Consequently, for fixed spheres, condition (7) is finally expressed as:

$0 \leqslant \zeta \leqslant \zeta_{\max }^{\text {fixed }}\left(\kappa\left(x_{\ell}\right)\right)$

Table 1

Calculated values of $\zeta_{\max }(k)$, and $\zeta_{\max }^{\text {fixed }}(k)$. For $k=7$ and 11 by interpolation. Last but one row for icosahedral. Last row for fcc and hcp

\begin{tabular}{rll}
\hline$k$ & $\zeta_{\max } /(a / 2)$ & $\zeta_{\max }^{\text {fixed }} /(a / 2)$ \\
\hline 1 & 1 & not applicable \\
2 & $\sqrt{3}$ & not applicable \\
3 & $\sqrt{2} \sqrt{3}$ & not applicable \\
4 & $5 \sqrt{3}$ & $\sqrt{3}$ \\
5 & $\sqrt{3} \sqrt{3}$ & $\sqrt{2} \sqrt{3}$ \\
6 & $\frac{4}{3} \sqrt{2} \sqrt{3}$ & $\frac{5}{3} \sqrt{3}$ \\
7 & $\sqrt{3} \sqrt{3}$ & $\sqrt{3} \sqrt{3}$ \\
8 & $2 \sqrt{2}$ & $2 \sqrt{2}$ \\
9 & $\sqrt{3} \sqrt{2}$ & $\sqrt{3} \sqrt{2}$ \\
10 & $\sqrt{3}$ & $\sqrt{3}$ \\
11 & 1 & 1 \\
12 ico & $\approx 0$ & $\approx 0$ \\
$12_{\text {fcc, hcp }}$ & 0 & 0
\end{tabular}

\section{2. $P_{k}(\zeta)$-the configuration distribution function}

A vector polygon formed by a chain of radial vectors according to Eq. (5), can be considered in terms of a random walk in space. We note that the steps of the walk are of the same size (Eq. (2)). If successive steps are represented by $R_{j}$, then the mean square displacement of a random walk is given by:

$\left\langle\zeta_{k}^{2}\right\rangle=\sum_{j=1}^{k} \sum_{i=1}^{k}\left\langle R_{j} \cdot R_{i}\right\rangle=k A(j, i)$,

with a correlation function defined as $A(j, i)=\left\langle R_{j} \cdot R_{i}\right\rangle /|R|^{2}$. The probability for the end-to-end vector being of magnitude lying in the range $(\zeta, \zeta+\mathrm{d} \zeta)$, equivalently, of lying in a shell of radius $\zeta$ and thickness $\mathrm{d} \zeta$, is given by

$P_{k}(\zeta) \mathrm{d} \zeta=4 \pi \zeta^{2} p_{k}(\zeta) \mathrm{d} \zeta$

subject to the normalizing condition that

$\int_{0}^{\zeta_{\text {max }}^{\text {fixed }}(k)} P_{k}(\zeta) \mathrm{d} \zeta=\int_{0}^{\zeta_{\max }^{\text {fixed }}(k)} 4 \pi \zeta^{2} p_{k}(\zeta) \mathrm{d} \zeta=1$,

where $P_{k}(\zeta)$ is a density function for the random variable, $\zeta$ and $p_{k}(\zeta)$ is a density function relating to spherical shells. In Eq. (10), $P_{k}(\zeta)$ is related to the function $\Phi_{k}\left(v_{1}, \ldots, v_{k}\right)$ by

$P_{k}(\zeta)=\int_{\left|v_{1}+\cdots+v_{k}\right|=\zeta} \Phi\left(v_{1}, \ldots, v_{k}\right) \mathrm{d} v_{1} \ldots \mathrm{d} v_{k}$.

Well known solutions to Eq. (10) exist for random unrestricted walks $[12,13]$. However, since the spheres are nonintersecting, the consecutive steps are subject to condition (3.1.2), leading to self-avoiding random walks (SARWs). Furthermore, the SARWs are limited by the finite space around the sphere's surface. We have shown above by geometrical calculations that the maximum end-to-end distance, i.e., the asymptotic limit for such SARWs decreases towards zero for $k \rightarrow 12$. The additional fixed sphere requirement limits the configurations of each cluster for $4 \leqslant k \leqslant 6$, such that $\zeta_{\max }$ cannot be reached and must be replaced by $\zeta_{\max }^{\text {fixed }} \leqslant \zeta_{\max }$. We note that $\zeta_{\max }^{\text {fixed }}=\zeta_{\max }$ for $k=7$ to 12 . There is approximate symmetry in the values of $\zeta_{\max }$ with respect to $k=6$, and in the values of $\zeta_{\max }^{\text {fixed }}$ with respect to $k=7$.

The function $P_{k}(\zeta)$ assumes a special and unique form for all clusters $(k=4, \ldots, 12)$ for which $\zeta=0$. For example, for equidistant angular configurations of points [14], $P_{k}(\zeta=0)=1$ by definition. Furthermore, clusters derived from Bravais lattices with $k=6,8$, and 12 (a subset of clusters with equidistant configurations) also have $\zeta=0$, and $P_{k}(\zeta)=1$.

Analytical solutions for the probability generating function (10) for self-avoiding walks of this type are not available at present [15]. Instead, we have carried out 200000 computer simulations of random clusters for each of $k=4$ to 10 , calculating the $\zeta$ value for each cluster and collecting the statistics. This can be achieved by placing points (on the surface of a unit sphere) defined in spherical coordinates by longitudinal angles 
Table 2

Fixed and loose sphere statistics for each of 200000 simulations of random clusters

\begin{tabular}{rrrr}
\hline$k$ & No. of fixed & No. of loose & \% fixed \\
\hline 4 & 87279 & 112721 & 43.64 \\
5 & 172649 & 27351 & 86.32 \\
6 & 198650 & 1350 & 99.33 \\
7 & 199997 & 3 & 99.99 \\
8 & 20000 & 0 & 100 \\
9 & 20000 & 0 & 100 \\
10 & 20000 & 0 & 100 \\
\hline
\end{tabular}

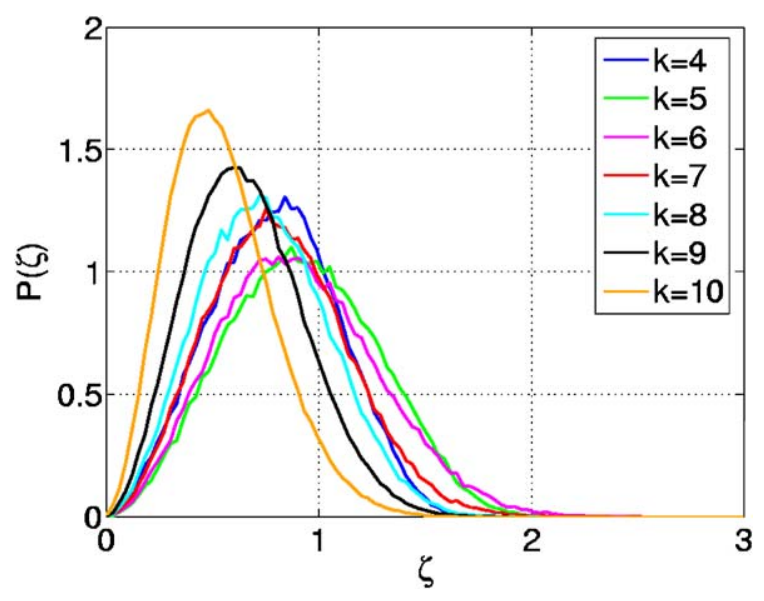

Fig. 3. Probability functions for the random variable, $\zeta$, from simulated random clusters with $k$ varying form 4 to 10 .

chosen between 0 and $2 \pi$ and latitudinal angles chosen between 0 and $\pi$. The points are created at random by independent and random selection of the angles. During simulation of a cluster, a point which violates condition (3.1.2) is rejected, and a new choice is made. The results for the fixed and loose configurations are shown in Table 2. Clearly, for $k=3$ (not simulated) all configurations would be in the loose category, whereas from $k=8$ increasing the probability of generating a loose configuration becomes insignificant. We shall return to this result later when predicting the coordination numbers. The accumulated statistical data for the $\zeta$ value for each cluster, excluding loose configurations, has been plotted in Fig. 3. It can be seen that the effect of self-avoidance in the limited space around the sphere forces the most probable displacement of the walk to decrease with increasing $k$ (for $k>7$ ). These results are complemented by the calculations shown in Table 1 . Indeed, for a close packed regular configuration of the outer spheres (fcc or hcp) the random walk must return to the starting point $(\zeta=0)$.

We note that the probability function, $P_{k}(\zeta)$, has both a descriptive character, as well as a predictive role. It describes the distribution of configurations for all clusters, as well as indicating the most probable configuration for each cluster. It is for this reason that we prefer this function to the angular measure, $s_{\beta}$, used previously $[9,16]$. In that sense, it is also more appropriate for describing configurations of random clusters in ideal amorphous solids than spherical harmonics [17]. The $Q_{6}$ component of spherical harmonics has a range of different values for regular configurations, but it gives a non-discriminating value of zero for random packings [18]. Only 2-dimensional plots of spherical harmonics differentiate between different configurations [19]. The function, $P_{k}(\zeta)$, however, gives a more comprehensive statistical description of the range of random configurations around the most probable arrangement with a clear differentiation between clusters with different coordinations ( $k$ values), and independent of the choice of frame of reference.

To the best of our knowledge, no published studies on selfavoiding random walks on the surface of a sphere have appeared previously, and therefore the results shown in Table 1 and Fig. 3 are new. Mathematicians are interested to find how many possible paths can be followed and what is the asymptotic behaviour for large number of steps. It is widely accepted that some answers are known for selected problems only and for a small number of steps [15]. In particular, for correlated walks (where each step depends on previous steps) the mean-square displacement is not linear in the number of steps $(k)$, as is evident from our results. However, the asymptotic behaviour for random walks on the surface of a sphere is clearly delineated: as $k \rightarrow 12, \zeta \rightarrow 0$ and $P_{k}(\zeta) \rightarrow 1$, with the width of the distribution reducing to zero.

\section{The coordination distribution $\{\Psi(k)\}$}

Consider a large finite spherical region, $\mathcal{S}$ say, in an ideal amorphous solid of infinite extent. Let $N$ denote the number of spheres with centres in $\mathcal{S}$, and $\mathbf{C}_{k}$ the number of such spheres with given coordination number $k$, where $k=4, \ldots, 12$. Then

$\sum_{k=4}^{12} \mathbf{C}_{k}=N$.

We could as easily regard $\mathcal{S}$ as being one of a sequence of such regions, indexed by $N$ say, and similarly $\mathbf{C}_{k}(N)$. Then the fraction of spheres with coordination number $k$ we would regard as satisfying

$\Psi(k)=\lim _{N \rightarrow \infty} \frac{\mathbf{C}_{k}}{N}=\Psi\left(\kappa_{\ell}\right)$,

i.e., the coordination number distribution $\{\Psi(k)\}$ coincides with the nearest-neighbour distribution and can be regarded as an asymptotic property of the solid.

$\Psi(k)=\mathbf{C}_{k} / N$.

We note that $\Psi(k)$ of Eq. (15) is directly related to the probability distribution for the nearest neighbours, as defined earlier:

$\Psi(k)=\Psi\left(\kappa_{\ell}\right)$.

From crystallography we know that for regular packing, for example body centered cubic, all spheres have the same coordination number $k=k_{\mathrm{bcc}} \equiv 8$. Then $\mathbf{C}_{8}=N$, and for the whole body

$\Psi(k)= \begin{cases}1, & k=k_{\mathrm{bcc}}, \\ 0, & \text { otherwise. }\end{cases}$

Similar results pertain to other regular packings in the sense that the distribution $\{\Psi(k)\}$ is degenerate (i.e., the coordination 
number of a randomly chosen sphere is the same irrespective of the sphere).

For a random packing we expect $\{\Psi(k)\}$ to be non-degenerate. One possible distribution comes from an argument initially proposed by Fürth [20] in connection with studies on the structure of liquids by Bernal [21]. Its concern is with packing arrangements subject to:

- coordination number constraints,

- the space available for spheres to occupy,

- the number of spheres that must be accommodated.

Start by noting that all spheres are of the same diameter and that there are no forces between them to cause preferential arrangements. The number of ways packing arrangements can occur depends on the space that can be occupied and the number of spheres available for packing. We describe this process via the following three variables:

(1) $k$, the number of nearest-neighbour spheres relative to each inner sphere;

(2) $x$, the maximum number of sites for spheres that can be in contact with the inner sphere; and

(3) $s$, the number of virtual sites available to spheres in a less dense packing.

In general $s \geqslant x \geqslant k$. Now from elementary combinatorics, $k$ spheres can be placed in $x$ sites in $\left(\begin{array}{l}x \\ k\end{array}\right)$ ways, and $x-k$ (empty) sites can be chosen from $s-x$ in $\left(\begin{array}{l}s-x \\ x-k\end{array}\right)$ ways, while $x$ sites can be distributed over $s$ sites in $\left(\begin{array}{l}s \\ x\end{array}\right)$ ways. The number of ways the $k$ spheres can be located amongst the $s$ sites subject to the maximum nearest-neighbour constraint, is thus

$$
\begin{aligned}
\Psi(k) \equiv \Psi(k ; s) & =\left(\begin{array}{l}
x \\
k
\end{array}\right)\left(\begin{array}{l}
s-x \\
x-k
\end{array}\right) /\left(\begin{array}{l}
s \\
x
\end{array}\right) \\
& =\frac{[x !(s-x) !]^{2}}{k ! s ![(x-k) !]^{2}(s+k-2 x) !} .
\end{aligned}
$$

Now assume that the number of sites is related to the available space around the inner sphere. Specifically, suppose that

$\frac{s}{v_{h}^{\prime \prime}}=\frac{x}{v_{h}^{\prime}}$,

where $v_{h}=V_{V}-v_{s}=v_{s}(1-p) / p, V_{V}$ if the volume of the Voronoï cell, $v_{s}$ is the volume of a sphere, and $p$ is the packing fraction. In relation (19) the prime corresponds to a special (close) packing with $s=x=k=12$ and a corresponding maximum density, and the double prime corresponds to a less dense random packing for which $s>x>k$, and the function, $\Psi(k)$, will have a non-degenerate distribution. Combining these relations with Eq. (19) gives a formula for $s$ :

$s=x\left(\frac{p^{\prime}\left(1-p^{\prime \prime}\right)}{p^{\prime \prime}\left(1-p^{\prime}\right)}\right)$.

For close packing we know that $x=12$ and $p^{\prime}=\pi / \sqrt{18} \approx$ 0.74 , whereas $p^{\prime \prime}$ for an IAS packing is not known a priori [22]. We believe that the frequently mentioned value of $p^{\prime \prime} \approx 0.64$
Table 3

Computed values of the $\Psi(k)$ function for the selected values of the parameter $s$ as shown

\begin{tabular}{lllllll}
\hline$k$ & $s=21$ & $s=21($ adj $)$ & $s=20$ & $s=19$ & $s=18$ & $s=17$ \\
\hline 1 & 0 & 0 & 0 & 0 & 0 & 0 \\
2 & 0 & 0 & 0 & 0 & 0 & 0 \\
3 & 0.000748 & 0 & 0 & 0 & 0 & 0 \\
4 & 0.015157 & 0.006780 & 0.003930 & 0 & 0 & 0 \\
5 & 0.097003 & 0.085863 & 0.050298 & 0.015718 & 0 & 0 \\
6 & 0.264060 & 0.268780 & 0.205380 & 0.128360 & 0.049774 & 0 \\
7 & 0.339510 & 0.347970 & 0.352080 & 0.330080 & 0.255980 & 0.127990 \\
8 & 0.212190 & 0.217500 & 0.275070 & 0.343830 & 0.399970 & 0.399970 \\
9 & 0.062872 & 0.064446 & 0.097801 & 0.152810 & 0.237020 & 0.355530 \\
10 & 0.008084 & 0.008286 & 0.014670 & 0.027507 & 0.053329 & 0.106660 \\
11 & 0.000367 & 0.000376 & 0.000762 & 0.001667 & 0.003879 & 0.009696 \\
12 & 0 & 0 & 0 & 0 & 0 & 0.000162 \\
sum $=$ & 1.000000 & 1.000000 & 1.000000 & 1.000000 & 1.000000 & 1.000000
\end{tabular}

is incorrect for reasons to be explained later, and instead we propose $p_{\text {IAS }}=p^{\prime \prime}=0.61$ as obtained empirically by extrapolation from previous simulation [16]. Substitution of $p^{\prime}=0.74$ and $p^{\prime \prime}=0.61$ in formula (20) gives $s \approx 21.8$. Taking the maximum whole number $s=21$, and $x=12$, formula (18) can be now evaluated. The result is as expected; a multi-valued function showing a preponderance of coordination numbers 6,7 and 8 as shown in Table 3, column 2. The Table also shows evaluations of (18) for $s=20$ through to 17 for reasons to be explained later.

To satisfy the IAS requirement that all spheres are in fixed positions, the predictions from formula (18) must be modified to take this into account. There are, possibly, a number of ways to do this.

Here, we take the simple approach of adjusting the predicted values shown in Table 3, column 2 by the fractions of fixed spheres derived from random simulation of clusters, as listed in Table 2, column 4. Thus, the values of $\Psi(k)$ for $s=21$ in Table 3 are first multiplied by the corresponding fraction (the fraction equals zero for $k=1,2,3$ and for $k=8, \ldots, 12$ ), and then increased proportionately so that the sum of all values equals 1.0 precisely. The adjusted values are shown in Table 3, column 3, and both sets of data are plotted on the graph in Fig. 4; for each $k$ the left-hand side columns (shaded) according to formula (18) for $s=21$, and the middle columns (solid) adjusted by the method described above. Furthermore, the righthand-side columns (shaded) are drawn from data obtained by computer generated Round Cell [16]. That the structure of the Round Cell is not identical with that of an IAS is corroborated by the differences in the distribution of coordination numbers shown in Fig. 4. There is an excessive proportion of clusters with $k=5$ and $k=6$ and reduced proportion with $k=8$ and $k=9$ as compared to the IAS, and this is reflected in the average coordination number calculated as 6.20 . Suffice it to say at this point that the packing of the Round Cell contains known flaws, and therefore it is not perfect in the IAS sense.

The characteristics of the amorphous body, including its density, are set by the relative proportions of the different clusters. The average coordination number for the IAS, calculated on the basis of data in columns 1 and 3 of Table 3 , is $\bar{k}=6.912$. In 


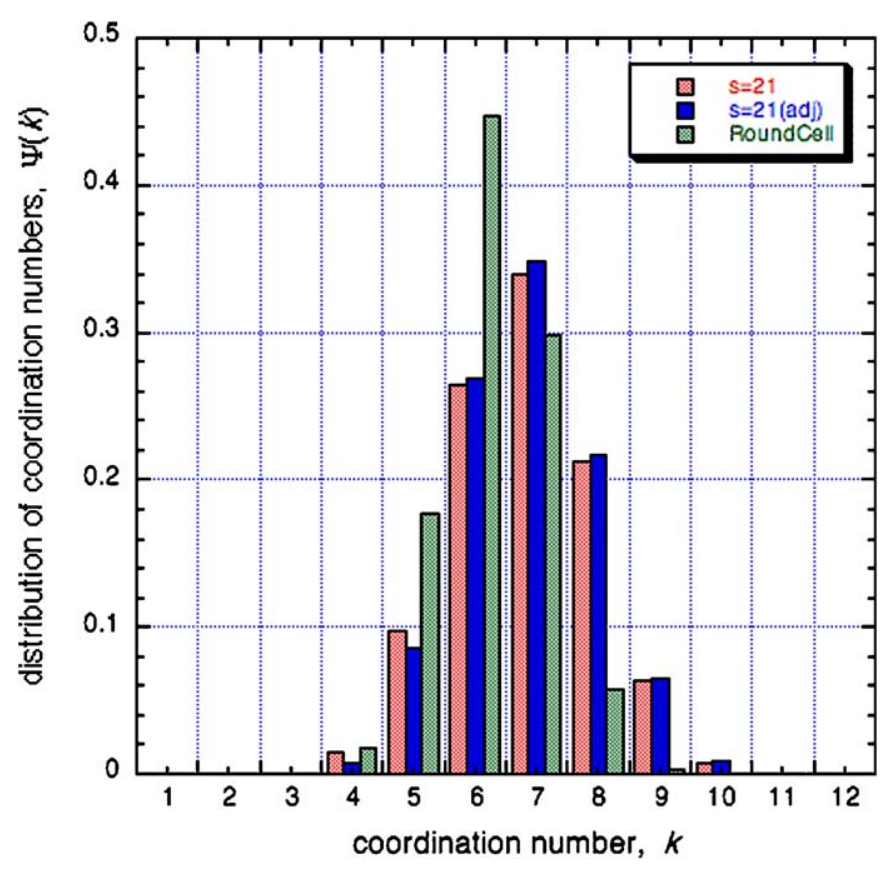

Fig. 4. Distributions of cluster coordination numbers: (i) shaded (red) columns-according to formula (18) for $s=21$, (ii) solid (blue) columns-the same as (i) but adjusted for fixed spheres only (see text), and (iii) shaded (green) columns-derived from Round Cell [16].

view of the distribution of coordination numbers one can imagine that clusters with $k$ values higher than $\bar{k}$ will be surrounded by clusters with $k$ values lower than $\bar{k}$, and vice versa, so that the full range of clusters is used, whilst density fluctuations across the body are localised.

In concluding this section, we propose that random amorphous packing of spheres of identical diameters is an ideal amorphous solid (IAS) if it is described by the statistical functions $P_{k}(k)$ and $\Psi(k)$ without exceptions. Since other ideal amorphous solids may well exist, we shall refer to this IAS as being of class I, and denote it as IAS-I.

Randomly packed spheres of two or three different sizes also form disordered structures [23], as do spheres each of different size [24,11], and it is expected that they will form separate amorphous classes. Since various other classes of random amorphous solids are possible, therefore, class I is reserved for amorphous solids based on single sized spheres for which the set $X$ (Eq. (1)) can be described by the functions $P_{k}(\zeta)$ and $\Psi(k)$ with $4 \leqslant k \leqslant 12$. IAS-I represents random packing of hard spheres, as well as random packing of chains consisting of linked hard spheres [9]. Packing of individual spheres is considered as a model of an amorphous monoatomic solid, and random packing of chains represents a model of an ideal amorphous polymer. In the latter case this would correspond to the so-called unified atom representation; this is an approach used frequently in modelling polymers.

\section{The imperfections in IAS-I}

Perfection of structure is defined firstly by the definition of imperfections that may occur, and secondly by the strict requirement of absence of those imperfections, namely no blem- ishes, flaws or defects of any kind, for which we shall use the collective term "flaws" (in contrast to "defects" used in crystalline solids).

For example, a gas in which the particle velocities conform precisely to the Maxwell distribution is referred to as an ideal gas; any deviation from this distribution is an imperfection. In the same sense, in an ideal amorphous solid the arrangement of spheres should conform precisely to distributions consistent with the functions $\Psi\left(\kappa\left(x_{\ell}\right)\right)$ and $\Phi_{k}(\zeta)$; any deviation is then an "imperfection".

Two general categories of flaws in the random amorphous structure can be defined: (i) geometrical and (ii) statistical, as described below.

\subsection{Geometrical (local) flaws}

We distinguish three kinds of geometrical flaws that can occur in the IAS-I structure:

(1) spheres of different sizes;

(2) loose spheres; and

(3) vacancies.

In an ideal amorphous solid composed of spheres of the same size, any sphere of a different size must be considered as a geometrical (and structural) flaw, as would be the case in ideal crystalline solids (substitutional defect). On a local level, such a different sphere may have its contact number outside the allowable range, depending on the size difference. On a larger scale, the effect of its presence would be evident (if in sufficient concentration) in the radial distribution function, or equivalently in the structure factor.

Loose spheres, called rattlers by Torquato and Stillinger [25], possess freedom of limited movement within the cage created by its neighbours. A quantitative measure of the movement of any sphere labelled $i$ say, is given by the value of the displacement, $J_{i}=\sqrt{u_{i}^{2}+v_{i}^{2}+w_{i}^{2}}$ [16], where $u_{i}, v_{i}$, and $w_{i}$ are components of the allowable movement $\mathbf{J}$ (in a Cartesian frame of reference) relative to its neighbours. By definition, for a fixed sphere, $J_{i}=\left|\mathbf{J}_{i}\right| \equiv 0$; for a loose sphere, $0<J_{i}<a$ because here we do not allow a vacancy. The definition of IAS-I requires that $\mathbf{J}_{i} \equiv 0$ for $i=1, \ldots, N$, i.e., all spheres are in fixed positions.

A vacancy is defined as an empty hole (unoccupied space) with its minimum dimension equal to or greater than the diameter of a sphere $\left(l_{\min } \geqslant a\right)$. It is considered as a serious flaw having a significant effect on the overall density of IAS. The condition that all spheres are fixed is sufficient to disallow the occurrence of vacancies in the IAS-I. An exception is the special case of clusters of 13 touching spheres with either fcc $(k=4+4+4)$ or hcp $(k=3+6+3)$ packing arrangement. In these cases removal of the central sphere would result in a vacancy with the surrounding spheres remaining in fixed (jammed) positions [7]. The occurrence of such clusters is considered to be a statistical flaw in the IAS-I structure, as described below. 


\subsection{Statistical (global) flaws}

It is possible to specify statistical flaws under two categories:

(1) flaws associated with the $\Psi(k)$ function; and

(2) flaws associated with the $P_{k}(\zeta)$ function.

With regard to the first category, a sphere with a contact number $k<4$ constitutes a flaw in the ideal amorphous structure. It is both a statistical flaw with respect to the $\Psi\left(\kappa\left(x_{\ell}\right)\right)$ function in that it violates the allowable range of values in the variable, and also a geometrical flaw in that it violates the requirement that all spheres be fixed. At the other end of the spectrum we note that in Euclidean space no sphere can have $k>12$. Such flaws cannot occur in any packing of spheres of identical diameter (physical impossibility) [26]. An important, and a more likely source of statistical flaws, is the departure from the ideal distribution of contacts predicted by the $\Psi\left(\kappa\left(x_{\ell}\right)\right)$ function. In a special packing, when the distribution becomes single-valued, e.g., $k=k_{\mathrm{bcc}}=8, \omega_{\Psi}=0$, the body will acquire regular structure, which may be perfectly ordered, or disordered, but it is no longer amorphous.

With regard to the second category, any arrangement of contact points on a sphere which corresponds to any of the five regular arrangements (Greek solids) is classified as a flaw because it represents an ordered (crystalline) structure and a departure from the prescribed distribution of contact points. More generally, any arrangement of contact points on a sphere which corresponds to equidistant angular configurations of points [14], or any of the Bravais lattices is classified as a flaw. Specifically, clusters of 13 spheres with hcp or fcc arrangement are considered as serious flaws in IAS-I, in that these increase drastically the average density of the body. It should be noted that an icosahedral arrangement of 13 spheres $(k=1+5+5+1)$ is not a flaw because in this case the touching neighbours can be moved around the inner sphere (whilst remaining in contact), hence $0 \leqslant \zeta \leqslant d$ (where $d$ is a constant), and the width of the distribution, $w_{P}>0$. Furthermore, removal of the inner sphere from an icosahedral cluster of 13 touching spheres (symmetrical or not) will make the outer spheres loose, and therefore it is not a special case, and not a statistical flaw.

\subsection{The effect of flaws on the density of IAS-I}

The transition from liquid-like to solid-like behaviour of randomly packed spheres is considered to occur at a packing fraction close to $0.49[27,28]$. This represents the lower limit for "solid" random packing of spheres. At the other end of the spectrum, the hcp or fcc arrangement of spheres represents the upper bound for sphere packing fraction at 0.74 . Therefore, the atomic packing fraction $p_{\text {IAS }}$ for IAS must lie within the range $0.49<p_{\text {IAS }}<0.74$ (more precisely defined by Torquato and Stillinger [22]). It is proposed for the model described here that its packing density is close to the value of 0.61 . It follows that random packings of spheres with densities higher or lower than that must contain flaws of the type described above. On the one hand, this view is supported by the results for simulated cells described elsewhere, see Fig. 4 in Ref. [16], in which an increasing number of vacancies and loose spheres was shown to lower overall density. On the other hand, higher density random packing models, approaching packing fraction of 0.64, show distinct splitting of the second peak in the radial distribution function [29,30], a clear indication of the presence of fcc or bcc clusters or their fragments, or alternatively a spiral connection of tetrahedra or local icosahedral packing [31,32].

Flaws vitiate the structure of the ideal amorphous solid, causing its density to vary from the hypothetical ideal value. Loose spheres and vacancies have the effect of lowering the density, whereas an undue presence of fcc and hcp clusters tends to increase density, both locally and globally. To support these assertions we analysed data from two separate publications in which the properties of simulated cells comprising large numbers of randomly packed spheres were described. First, "Round Cells" comprising randomly packed spheres around a central nucleus were created by computer simulation [16]. An algorithm was devised to add spheres to an existing surface, starting at the nucleus. Round Cells of different densities were obtained in one of two ways: (i) specific parameters in the algorithm were adjusted to reduce optimum packing; and (ii) spheres were removed at random from a given cell of maximum density. Fig. 5 shows the ensuing relationship between the packing densities obtained and the content of loose spheres in the Round Cells. As expected, global packing density decreases as the fraction of loose spheres increases. A line fitted to the data points, starting at 0.49 and $80 \%$ loose sphere content

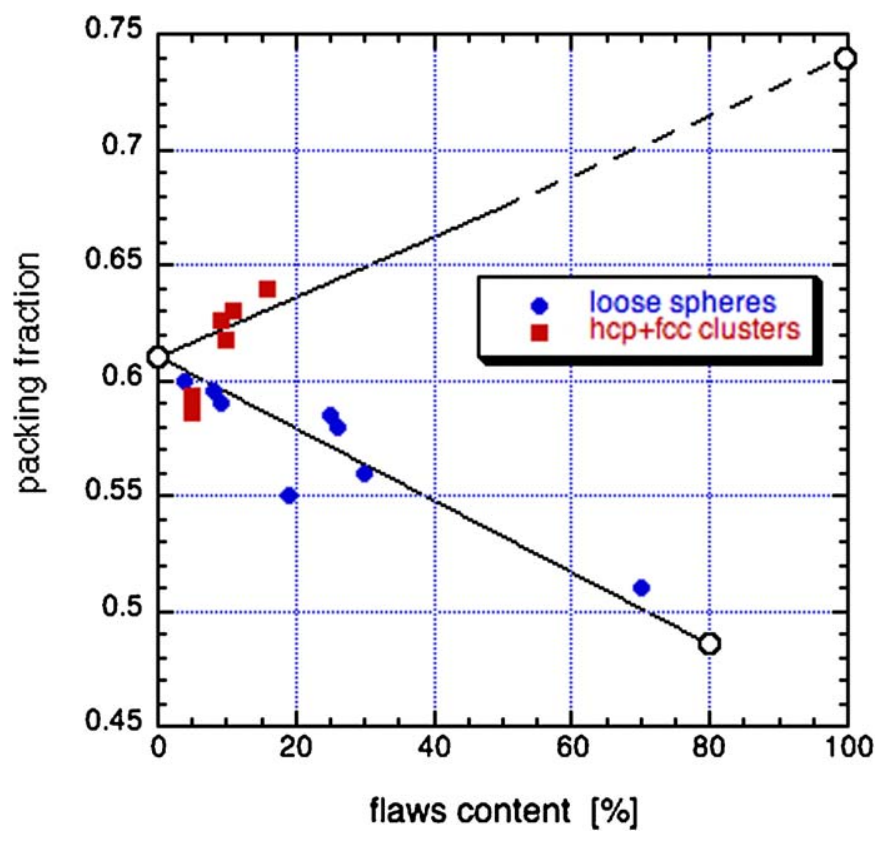

Fig. 5. Density versus content of flaws for random packings: (i) square points (red) - combined fraction of hcp and fcc flaws in experimental random packings [33], and (ii) round points (blue)-loose sphere content in simulated Round Cell [16]. Lines drawn from predicted packing fraction of IAS (0.61) to close packing $(0.74)$, and predicted packing fraction of IAS (0.61) to liquid/solid transition (0.49) [27]. The upper line appears broken at high flaw content to indicate that amorphous structure may change its nature and become disordered structure as it approaches the ideal crystalline state. 
(solid-liquid transition point), and extrapolated to zero content of loose spheres points to a value close to 0.61 for the density of a packing containing none of these flaws. We propose that the Round Cell with the maximum packing density $(0.603)$ is a close approximation of IAS-I, and that the most likely packing density of IAS-I is close to that value, significantly removed from the "typical" value of 0.64 [6].

In another publication, "Cylindrical Cells" were constructed by pouring a large number of hard spheres into a container whilst holding a stick in the middle of it; the stick was then removed, leaving a packing density of approximately 0.58 [33]. Higher densities, up to approximately 0.64 , were achieved by gently tapping the sample. The packings were subjected to Xray tomography and computational rendering of the spheres, so that geometrical properties of the packings could be measured. In particular, the results showed significant fractions of fcc and hcp clusters occurring in the samples. The data in Table 2 from Ref. [33] are also plotted in Fig. 5, illustrating the relationship between the packing density and the combined fractions of fcc and hcp clusters. In this case the global packing density of samples increases with the number of high density clusters which are considered as both geometrical and statistical flaws in the IAS-I. This is a remarkable observation in that we are accustomed to thinking of defects as always decreasing the density of crystalline materials, whereas a flaw in an ideal amorphous solid evidently can have either positive or negative effect on density, depending on the nature of the flaw. It is supposed that the two red square points at 5\% flaw content, but well below 0.6 packing fraction, must correspond to Cylindrical Cells that retained the large number of loose spheres and vacancies expected from the method of construction, with a small content of fcc and hep clusters formed by gentle tapping. It is assumed that in these Cells the presence of loose spheres and vacancies is the dominant flaw type causing overall reduction in packing density.

Now, consider the effect of short and long range "order" as factors in density variations. By definition, short range order in solids refers to ordered packing of atoms in small volumes, ranging over several atomic distances. Ordered packing in a (say, convex) region means that one or more symmetry elements should be found in the arrangement of atoms confined within that region. For any random cluster to exhibit short range order in itself, it would have to possess a minimum of one statistical geometry element [9]. Examples of short range order in real materials include

- (i) Guinier-Preston (GP) zones in Al-Cu alloys [34];

- (ii) Cationic short range order in crystalline ionic conductor [35];

- (iii) i-phase nano-crystals in $\mathrm{Zr}$ based bulk metallic glass (BMG) [36].

The GP zones form in the shape of a disc with translational symmetry of $\mathrm{AlCu}_{2}$ atomic groups along its basal plane and rotational symmetry perpendicular to its base. The second example refers to supercell structure within a Hollandite crystal consisting of $\mathrm{TiO}_{6}$ and $\mathrm{MgO}_{6}$ octahedra within tetragonal unit cell. The third example represents near spherical particles with icosahedral configuration (pseudocrystalline) appearing in the 5 element metallic alloy with random amorphous atomic packing.

Is short range "order" possible in the random packing of spheres, and specifically, in an ideal amorphous solid? We can disqualify compositional variation since all spheres are identical, leaving variation in packing arrangement as the only source of differentiation. Each cluster can be described by (at least) three statistics:

- the number of contact points $k$;

- the configuration of the nearest neighbours of the inner sphere, described by $\zeta$; and

- the orientation of the cluster in space, described by the measure $\Omega$.

For two clusters ( 1 and 2 ) to be identical we should necessarily have

$k_{1}=k_{2}, \quad \zeta_{1}=\zeta_{2}, \quad \Omega_{1}=\Omega_{2}$.

Each cluster has 9 possible values for $k=4, \ldots, 12$. If the distribution for $k$ is equiprobable then the probability that two clusters have the same number of contact points (the same value of $k$ ) is $\sum_{k=4}^{12}\left(\frac{1}{9}\right)^{2}=\frac{1}{9}$. For any given distribution $\Psi_{k}$ as in Section 4 then we can write more generally that the probability for two clusters to have the same value of $k$ is $\sum_{k=4}^{12}\left[\Psi_{k}\right]^{2}=\frac{1}{9} c_{1} \geqslant$ $\frac{1}{9}$, where $1 \leqslant c_{1}<9$ is some constant.

For the other two variables we note that two independent copies of random variables with continuous distributions agree with probability 0 . Therefore, the probability that two or more neighbouring clusters have identical coordination and configuration and are oriented in precisely the same way, is zero. Repeatability of an ordered or regular pattern in adjacent clusters extending along a line is zero. Consequently, it can be stated with generality that the body is homogeneous and isotropic down to a certain course-grain measure that can be identified as the representative volume element [9].

\section{Discussion and conclusions}

\subsection{Construction of IAS}

Haüy's work on the structure of solids showed that regular three-dimensional stacking of equal sized balls leads to crystalline order. He constructed models of single crystals with specific crystal faces to be identified later by the law of rational indices [37]. Given the description of random packing presented in this paper, one approach to construct a model of IAS-I could be by Haüy's method as follows. Start with $N$ spheres segregated into $\mathbf{C}_{k}$ collections, with populations defined by formula (18), and listed in Table 3. Within each collection the spheres are marked with contact points such that the configurations of the points conform to the distributions shown in Fig. 3. Next, spheres are sampled from the collections with frequencies proportional to their content, and fitted onto a growing cluster by matching their contact points. Clearly this is a tedious 
process, since the spheres are not numbered and the sequence of stacking is not known a priori. An alternative approach would be to define geometrically the point set $X$, and then affix spheres centred at all the points specified by the set.

The sequential approach is more amenable to the task being carried out by computer in a way similar to the construction of Round Cells reported in a previous publication [16]. To ascertain the success of such simulations, the Round Cell with maximum packing density (0.603) and a minimum content of flaws $(<4 \%)$ was subjected to computation of the cluster configurations, expressed in terms of relation (10), and distribution of nearest neighbours expressed in terms of relation (18). The results for the distribution of nearest neighbours are shown in Fig. 4 by shaded (red) columns, adjacent to the results for the IAS-I calculated theoretically, and shown as solid (blue) columns. There is broad qualitative agreement between the two distributions, in that both show multiple peaks with a similar trend in the frequency of clusters with the corresponding contact numbers. However, the data for the Round Cell shows greater numbers of clusters with lower coordination numbers, as would be expected for a body with lower packing density $(\approx 0.603)$ than that of IAS-I $(\approx 0.61)$. This expectation is based on the assumption that clusters with higher coordination numbers have smaller Voronoï volumes and greater packing density, as described in a separate publication [38]. Both distribution functions show almost total absence of clusters with $k=11$ and 12 .

Neglecting the small number of loose spheres in the Round Cell, we have calculated and plotted in Fig. 3 the distribution functions, $P_{k}(\zeta)$ of the length $\zeta$ of the closing vector of contact points (see Eqs. (5) and (6)) for fixed sphere clusters with $k=4$ to 9 . In Figs. 3 and 6 these simulations come from the independent random clusters generated as in Section 3, and the clusters from the Round Cell created by computer generated random packing spheres [16], respectively. The similarity of the shape of the functions provides tangible evidence supporting the view that the construction of the Round Cell according to an algorithm described in the previous paper is a process

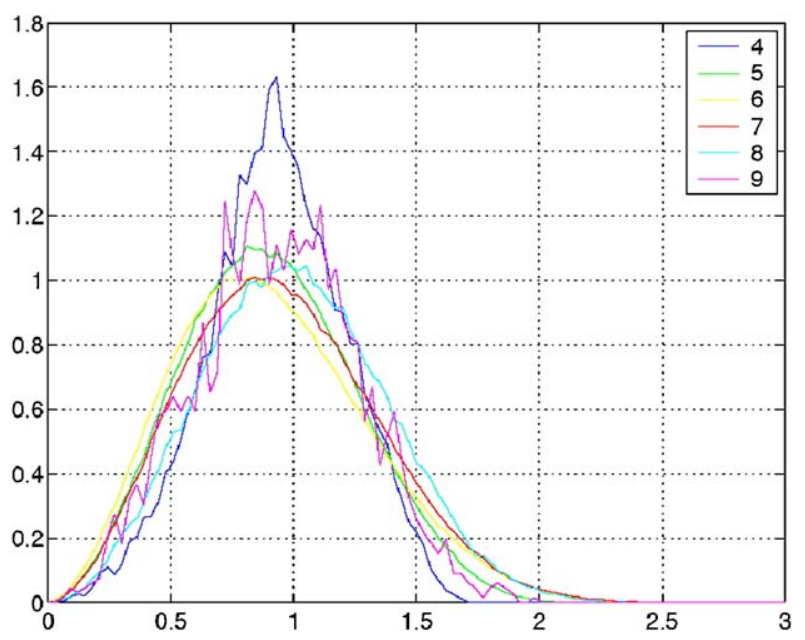

Fig. 6. Probability functions for the random variable, $\zeta$, derived from simulated Round Cell with packing density (0.603) [16]. leading to random packing similar to the one described in this paper for IAS. The poorer quality of the plots in Fig. 6 is simply due to smaller sample sizes obtained from the Round Cell $\left(10^{5}\right.$ spheres), compared to the random cluster simulations $\left(2 \times 10^{5}\right.$ spheres for each $k=4, \ldots, 10)$. Also, comparison between the two sets of data should be made mainly for $k=5$ to 8 since the data for the Round Cell are very limited for $k$ values outside this range (see again Ref. [16]).

The shifting position of the peaks in Fig. 3, at first moving away from $\zeta=0$ (for $k=4$ and 5), and then towards $\zeta=0$ (for $k>6$ ) is an effect expected from the geometrical limitations of the SARWs. In Fig. 6 it is hard to discern whether such a trend is also present. To measure the difference between the two, the comparative cumulative distribution functions (CDFs) are plotted in Fig. 7. In the first two plots $(k=5$ and 6$)$ the CDFs are overlapping, but clear differences between the CDFs appear for $k=7$ and 8 . We computed the Kolmogorov-Smirnov test statistic $D$ [39] for the two distributions. To calculate $D$, the CDFs of the distances $\zeta$ are normalized by dividing each by its mean value. Then $D$, the maximum value of the absolute difference of the normalized CDFs, and $Q_{\mathrm{KS}}$, the significance level of an observed $D$ value, were calculated and are shown in Table 4. Since differences were found between the statistical characteristics of the simulated random clusters and those of the clusters derived from the Round Cell of randomly packed spheres, we conclude that the latter is not a perfect amorphous solid, although it may be a crude approximation of it.

\subsection{Contact configuration function}

Changing the value of the integer parameter $s$ in Eq. (18) leads to different evaluations of the $\Psi(k)$ function. Not all values need be considered; if the density of the solid is not to exceed 0.64 , then values $s \leqslant 18$ are disqualified. On the other hand, values of $s>21$ result in a density which is too low, which leaves 3 values: $s=21,20$ and 19. At this stage we have no additional physical arguments for $s=21$ in preference to $s=20$ or $s=19$, other than the hypothetical link to density through relation (20). Although it appears that there may be three variants, namely IAS-Is21, IAS-Is20 and IAS-Is 19, corresponding to the three values of the parameter, $s$, respectively, yet intuitively we feel that a unique solution is more likely. This view is supported by the work of Edwards and coworkers $[40,41]$, who postulate a statistical mechanics approach to jammed [7] configurations. In particular, they develop the concept of entropy of microcanonical ensemble of jammed configurations expressed as follows [40]:

$S=\log \int \delta(V-W(\varsigma)) \Theta(\varsigma) \mathrm{d} \varsigma$

where $\delta$ is the Heaviside function, $V$ is the volume of the body of $N$ spheres (analogous to the internal energy of a system), $\varsigma$ represents the collective coordinates of contact point positions in the static packing, and $W(\varsigma)$ is a volume function expressed in terms of contact variables (analogous to the Hamiltonian of a system in statistical mechanics). According to Ref. [40], under the condition, $V=W$, the system is said to be "jammed", and the function $\Theta(\varsigma)$ ensures that all spheres 

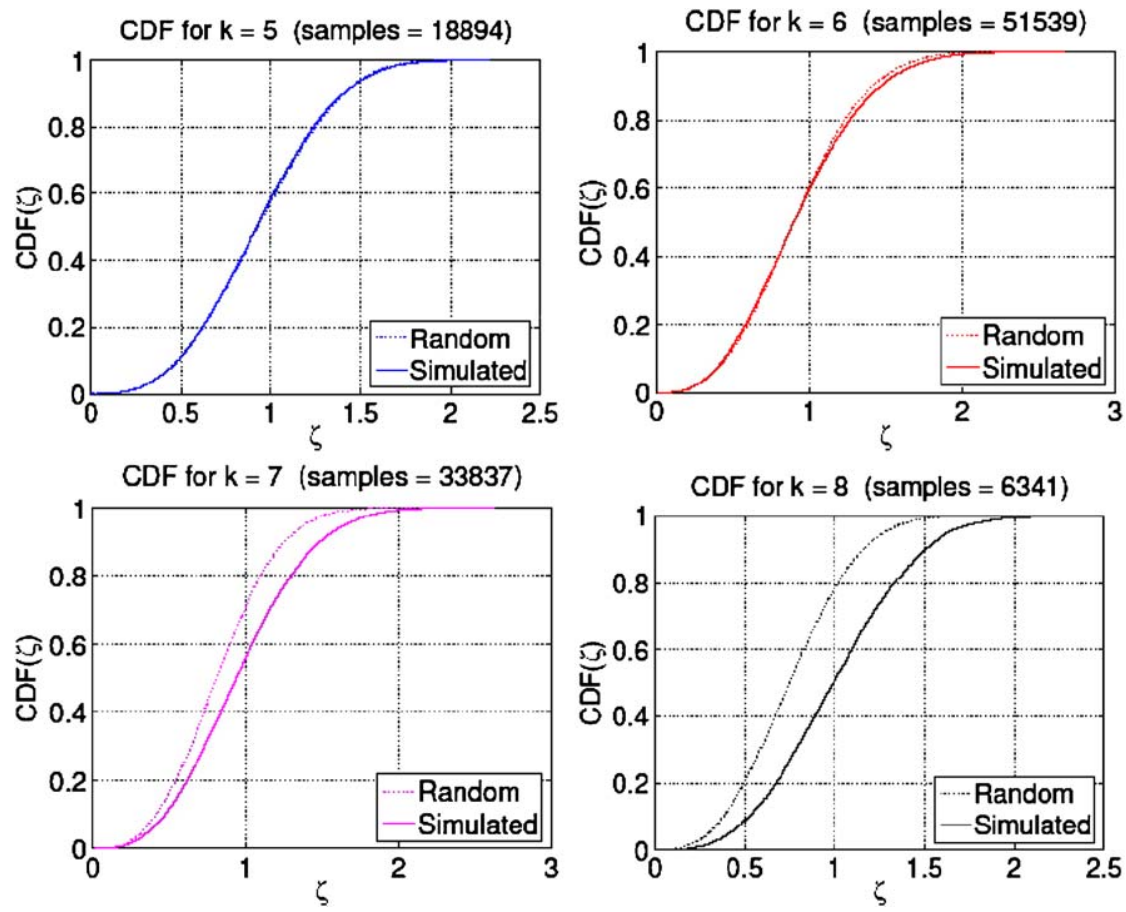

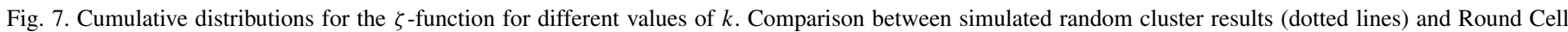
(solid lines) [16] using Kolmogorov-Smirnov analysis [39].

Table 4

Computed values of the Kolmogorov-Smirnov statistic test between the simulated random clusters (Fig. 3) and clusters taken from the Round Cell (Fig. 6)

\begin{tabular}{|c|c|c|c|c|c|c|c|c|}
\hline \multirow[t]{2}{*}{$N_{\text {sample }}$} & \multicolumn{2}{|l|}{$k=5$} & \multicolumn{2}{|l|}{$k=6$} & \multicolumn{2}{|l|}{$k=7$} & \multicolumn{2}{|l|}{$k=8$} \\
\hline & $D$ & $Q_{\mathrm{KS}}(D)$ & $D$ & $Q_{\mathrm{KS}}(D)$ & $D$ & $Q_{\mathrm{KS}}(D)$ & $D$ & $Q_{\mathrm{KS}}(D)$ \\
\hline 10000 & 0.01414 & $5.23 \mathrm{e}-002$ & 0.0237 & $7.15 e-005$ & 0.1549 & $2.40 \mathrm{e}-190$ & & \\
\hline 1000 & 0.02258 & $6.89 \mathrm{e}-001$ & 0.04865 & $1.78 \mathrm{e}-002$ & 0.1609 & $7.09 \mathrm{e}-023$ & 0.2896 & $4.10 \mathrm{e}-073$ \\
\hline 100 & 0.1402 & $3.55 \mathrm{e}-002$ & 0.06351 & $8.03 \mathrm{e}-001$ & 0.228 & $4.68 \mathrm{e}-005$ & 0.329 & $4.61 \mathrm{e}-010$ \\
\hline 10 & 0.152 & $9.61 \mathrm{e}-001$ & 0.1426 & $9.79 \mathrm{e}-001$ & 0.2646 & $4.24 \mathrm{e}-001$ & 0.3835 & $7.86 \mathrm{e}-002$ \\
\hline
\end{tabular}

are in fixed (locked) positions. One can infer from this condition that the system will achieve a unique state in terms of its density, this in turn implying a unique value of the parameter $s$.

We suspect that it may not be possible to construct an IAS by any experimental method, just as it is effectively impossible to grow an ideal, completely defect free crystal. Certainly, all experimental attempts at random packings of spheres suffer at least three limitations that prevent even a close approximation to IAS. First, the force of gravity: its effect during the dynamic stage of pouring causes some clusters of spheres to arrange into locally close packed configurations (fcc, hcp), or to rearrange into these at a later stage by tapping or vibration. Second, friction: it limits the freedom to form random configurations and it is likely to be responsible for loose spheres in the structure. Third, inhomogeneity: this results from the directionality of pouring of the spheres into a container, and also from edge effects due to the presence of the container walls. All three limitations can be avoided by computer simulation of Round Cells, as described in Ref. [26, p. 166], and in Ref. [16].

\subsection{AIS as a model of real amorphous solids}

Finally, to understand better the structure of real amorphous materials in terms of observable and measurable differences between the ideal and the real, one should consider the range of materials to which the IAS-I may be applicable. Besides being purely monoatomic, the materials must have the property of non-directional interatomic bonding so as to allow for the range of coordination numbers displayed by the IAS-I. Furthermore, they must have the ability to condense into an amorphous glassy solids. On the one hand, elements of group IB and VIIIA are obvious candidates if only their crystallisation could be suppressed. The amorphous structure of argon has been studied [42]. Modelling of sodium [30] and aluminium [43] has also been tried. On the other hand, the IAS-I is not appropriate for conventional glasses $\left(\mathrm{MO}_{2}\right)$ which have coordination numbers limited to the range from 3 to 5 [44]. Similar comment applies to amorphous silicon and amorphous carbon [45]. The IAS-I may be a suitable model for amorphous polymers if their structure can be simplified to a unified atom representation, although this will have to be tested and verified. Finally, bulk amorphous 
metals [46] represent a new class of amorphous structures with a wide distribution of coordination numbers for which a model based on random packing of spheres of 5 different sizes [47] could be an extension of the IAS approach.

\subsection{Brief conclusions}

(1) In geometrical terms amorphous solids are fundamentally different from crystalline solids in that they cannot be constructed by the crystallographic method of translation of the basis along the lattice points. They do not possess any lattice.

(2) A random structure is not merely a disordered structure. A disordered crystal structure can be gradually restored to its fully ordered state by removal of its defects. In principle, an amorphous structure with flaws can be rearranged to its ideal (perfectly random) state.

(3) The IAS-I is an irregular assemblage of spheres containing no imperfections. By definition, it shows absence of preferred local configurations and absence of local flaws. It is of infinite extent (no edge effects) and shows no orientation. It is homogeneously random (it is ergodic with respect to detail of local patterns).

(4) Flaws in amorphous solids can increase or decrease average body density, depending on the type of flaw.

(5) The mean coordination number of spheres is rational in an ideal crystalline structure, and hypothesised to be irrational in an ideal amorphous solid.

\section{Acknowledgement}

One of us (Z.H.S.) acknowledges financial assistance from the ANU Centre for the Science and Engineering of Materials (CSEM).

\section{References}

[1] C.P. Flynn, Point Defects and Diffusion, Clarendon Press, Oxford, 1972.

[2] A.M. Walker, B. Slater, J.D. Gale, K. Wright, Nature materials, advance online publication, http://www.nature.com/naturematerials, 2004.

[3] A.H. Cottrell, Dislocations and Plastic Flow in Crystals, Clarendon Press, Oxford, 1958.

[4] J. Gittus, Creep, Viscoelasticity and Creep Fracture in Solids, Appl. Sci. Publ., London, 1975.

[5] I. Turek, Electronic Structure of Disordered Alloys, Surfaces and Interfaces, Kluwer Academic Publishers, Boston, 1997.

[6] T.M. Truskett, S. Torquato, P.G. Debenedetti, Phys. Rev. E 62 (2000) 993.

[7] S. Torquato, T.M. Truskett, P.G. Debenedetti, Phys. Rev. Lett. 84 (10) (2000) 2064.

[8] Ch. Zong, Sphere Packings, Springer-Verlag, New York, 1999.

[9] Z.H. Stachurski, Phys. Rev. Lett. 90 (15) (2003) 5502.
[10] J.N. Conway, N.J.A. Sloane, Sphere Packings, Lattices and Groups, third ed., Springer-Verlag, New York, 1998.

[11] D.J. Daley, Packings and approximate packings of spheres, NISSG No DMS-9313013.

[12] M. Barber, B. Ninham, Random and Restricted Walks, Gordon and Breach, New York, 1970.

[13] L.R.G. Treloar, The Physics of Rubber Elasticity, Clarendon Press, Oxford, 1975.

[14] L.L. Whyte, Unique arrangement of points on a sphere, The American Mathematical Monthly 59 (9) (1952) 606.

[15] N. Madras, G. Slade, The Self-Avoiding Walk, Birkhäuser, Boston, 1993.

[16] L.-Th. To, Z.H. Stachurski, J. Non-Cryst. Solids 333 (2004) 161.

[17] P.J. Steinhardt, D.R. Nelson, M. Ronchetti, Phys. Rev. B (1983) 805.

[18] T. Gruhn, P.A. Monson, Phys. Rev. E 64 (2001) 061703.

[19] T. Aste, M. Saadatfar, T.J. Senden, Phys. Rev. E 71 (2005) 061302.

[20] R. Fürth, A new approach to the statistical thermodynamics of liquids, Proc. Roy. Soc. Edinburgh 66 (1964) 232.

[21] J.D. Bernal, Proc. Roy. Soc. Lond. A 280 (1964) 299.

[22] S. Torquato, F.H. Stillinger, J. Phys. Chem. B 106 (2002) 8354.

[23] J. Villain, K.Y. Szeto, B. Minchau, W. Ren, in: D.P. Landau, K.K. Mon, H.-B. Schüttler (Eds.), Computer Simulation Studies in Condensed Matter Physics III, Springer-Verlag, Berlin, Heidelberg, 1991.

[24] D.L. Weaire, S. Hutzler, The Physics of Foams, Clarendon Press, Oxford, New York, 1999.

[25] S. Torquato, F.H. Stillinger, J. Chem. Phys. B 105 (2001) 11849.

[26] D. Stoyan, W.S. Kendall, J. Mecke, Stochastic Geometry and its Applications, Wiley, New York, 1995.

[27] H. Reiss, A.D. Hammerich, J. Phys. Chem. 90 (1986) 6260.

[28] N.P. Kopsias, D.N. Theodorou, J. Chem. Phys. 109 (19) (1998) 8573.

[29] S. Torquato, Random Heterogeneous Materials, Springer-Verlag, New York, 2002.

[30] M.S. Watanabe, K. Tsumaraya, J. Chem. Phys. 87 (8) (1987) 4891.

[31] K. Tsumuraya, M.S. Watanabe, J. Chem. Phys. 92 (8) (1989) 4983.

[32] F. Spaepen, Nature 408 (2000) 781.

[33] T. Aste, M. Saadatfar, A. Sakellariou, T.J. Senden, Physica A 339 (2004) 16.

[34] T.J. Konno, M. Kawasaki, K. Hiraga, J. Electron. Microsc. (Tokyo) 50 (2) (2001) 105 .

[35] H.U. Beyeler, Phys. Rev. Lett. 37 (23) (1976) 1557.

[36] G. Wang, J. Shen, J.F. Sun, B.D. Zhou, J.D. FitzGerald, D. Llewellyn, Z.H. Stachurski, Scripta Materialia 53 (2005) 641.

[37] G. Friedel, Leçons de Cristallographie, Blanchard, Paris, 1964.

[38] L.-Th. To, Z.H. Stachurski, J. Non-Cryst. Solids (2005), in press.

[39] W.H. Press, S.A. Teukolsky, W.T. Vetterling, B.P. Flannery, Numerical Recipes in C: The Art of Scientific Computing, Cambridge Univ. Press, Cambridge, 1992.

[40] S.F. Edwards, D.V. Grinev, Adv. Phys. 51 (8) (2002) 1669.

[41] C.C. Mounfield, S.F. Edwards, Physica A 210 (1994) 279.

[42] J. Farges, M.F. de Feraudy, B. Raoult, G. Torchet, J. Chem. Phys. 78 (8) (1983).

[43] R.-S. Liu, K.-J. Dong, J.-Y. Li, A.-B. Yu, R.-P. Zou, J. Non-Cryst. Solids 351 (2005) 612.

[44] W.H. Zachariasen, J. Chem. Soc. 54 (1932) 3841.

[45] H. Fritzsche (Ed.), Amorphous Silicon and Related Materials, World Scientific, Singapore, 1989.

[46] A. Inoue, Acta Mater. 48 (2000) 279.

[47] S.I. Henderson, T.C. Mortensen, S.M. Underwood, W. van Megen, Physica A $233(1996) 102$. 